Intensivmed 2009 · 46:464-465

DOI 10.1007/s00390-009-0091-7

Online publiziert: 2. Oktober 2009

(c) Springer-Verlag 2009

\section{T. Welte}

Klinik für Pneumologie, Medizinische Hochschule Hannover

\section{Back to the roots}

\section{Stehen wir vor einem neuen Zeitalter der Infektionskrankheiten?}

Seit Wochen dominiert die Debatte um ein neues Influenza-Virus, $\mathrm{H}_{1} \mathrm{~N}_{1}$, die öffentliche Diskussion weltweit. Nun scheint das Virus wesentlich weniger pathogen als befürchtet, schwere Krankheitsverläufe stellen eine Rarität dar. Auch eine Verschmelzung des $\mathrm{H}_{1} \mathrm{~N}_{1}$-Virus mit klassischen Influenzaviren, von der eine Zunahme der Pathogenität befürchtet worden war, blieb bisher aus. Die Erfahrungen auf der Südhalbkugel, v. a. in Australien und Südamerika, geben momentan nicht zu Befürchtungen Anlass. Der Erreger des schweren Atemnotsyndroms (SARS) 2003 oder $\mathrm{H}_{5} \mathrm{~N}_{1}$, verantwortlich für die sog. Vogelgrippe, hatten eine ausreichend pathogenes Potenzial, beide waren jedoch glücklicherweise - aus unterschiedlichen Gründen - nicht in der Lage, eine Pandemie auszulösen.

Die wirklichen Probleme, die solche neuen Erreger mit sich bringen könnten, werden jedoch leider in der Öffentlichkeit wenig thematisiert. Selbst in einem so gut organisierten Land wie Deutschland sind wir nicht wirklich auf einen Massenanfall von Kranken eingestellt. Wie soll die Betreuung der Patienten erst in Südeuropa oder gar in den Entwicklungsländern gewährleistet werden? Die Globalisierung ermöglicht heute eine Erregerausbreitung über die ganze Welt in kürzester Zeit, Pandemien werden in Zukunft eher die Regel als ein Einzelereignis darstellen.

Ein Impfstoff konnte im Falle von $\mathrm{H}_{1} \mathrm{~N}_{1}$ und $\mathrm{H}_{5} \mathrm{~N}_{1}$ in kurzer Zeit entwickelt werden, weil die Erfahrungen aus der Entwicklung der klassischen Influenzaviren genutzt werden konnten, aber was würde passieren, wenn eine Pandemie mit einem Nicht-Influenza-Virus bevorstünde. Am
Beispiel des HI-Virus kann man nachvollziehen, welche Schwierigkeiten sich schnell verändernde Oberflächenstrukturen bei der Impfstoffentwicklung bereiten können.

Mit den Neuraminidasehemmern steht zur Behandlung der Influenza zumindest eine antivirale Substanz zur Verfügung, auch wenn im Falle $\mathrm{H}_{1} \mathrm{~N}_{1}$ die Wirksamkeit dieses Medikamentes nicht wirklich geklärt ist und weltweit zunehmend Resistenzen zu beobachten sind. Für andere Viren fehlt - wie man bei SARS gesehen hat - jede therapeutische Alternative. Dazu kommt, dass viele Patienten gar nicht an der eigentlichen Viruserkrankung versterben, sondern eine sekundäre bakterielle Infektion zum Tode führt. Der Zusammenhang zwischen Influenzainfektion und sekundärer Pneumokokkenoder Staphylokokkenbakteriämie ist gut bekannt. Gegen alle wichtigen bakteriellen Erreger haben wir jedoch seit Jahren steigende Resistenzraten weltweit. Demgegenüber kommen immer weniger neu entwickelte Antibiotika auf den Markt. Viele pharmazeutische Firmen investieren nicht mehr in die Antibiotikaentwicklung, weil sie darin kein Gewinnpotenzial mehr erkennen. Infektionen mit bakteriellen Erregern, bei denen keine der bekannten antiinfektiven Substanzen mehr wirkt, sind keine Seltenheit. Schon sprechen Experten vom postantibiotischen Zeitalter.

Welche Konsequenzen müssen wir aus dieser Entwicklung - dem immer schnelleren Auftauchen und der rasanten Ausbreitung neuer Erreger bei gleichzeitigem Stagnieren der Antiinfektivaentwicklung - ziehen?
Zweifellos muss die Forschung im Bereich der Infektiologie - gerade auf dem Gebiet der Arzneimittelentwicklung und der Vakzinierung - verstärkt werden. Ergebnisse sind jedoch nicht kurzfristig zu erwarten. Der Infektionskontrolle, deren wesentliche Aspekte in diesem Heft von S. Scheithauer und S.W. Lemmen dargestellt werden, kommt hierbei eine entscheidende Bedeutung zu.

Ergänzend dazu muss jedoch die infektiologische Ausbildung von Ärzten verbessert werden. Der Antibiotikakonsum unserer Bevölkerung ist gewaltig, häufig werden Substanzen nicht indikationsgerecht eingesetzt. Die Angst des Behandlers, etwas $\mathrm{zu}$ übersehen, führt $\mathrm{zu}$ viel zu langer und oft falscher Antibiotikaverschreibung. Gute, in der Praxis umsetzbare Leitlinien können eine Richtlinie in der täglichen Praxis sein. Die S3-Leitlinie zur Behandlung der unteren Atemwegsinfektion und der ambulant erworbenen Pneumonie ist ein Musterbeispiel einer gelungenen Empfehlung. In diesem Heft stellt G. Höffken als Sprecher der Leitliniengruppe die für die schwere Atemwegsinfektion verbindlichen Empfehlungen vor.

Besonders schwierig ist die Situation für den Arzt am Patientenbett, wenn evidenzbasierte Leitlinien fehlen. Das gilt umso mehr bei wichtigen, aber eher selten auftretenden Krankheitsbildern. C. Eckmann und H. Schmidt et al. stellen den aktuellen Kenntnisstand für die schwere Haut- und Weichteilinfektion bzw. die Meningitis zusammen und geben damit eine Orientierung für die tägliche Praxis.

Der Beitrag zur Infektiologie in diesem Heft wurde von einer Infektionsepidemi- 
ologin, einem Pneumologen, einem Neurologen und einem Chirurgen verfasst. Hier spiegelt sich die Interdisziplinarität der Infektiologie wieder. Nur durch Zusammenarbeit vieler Fachgebiete wird es in Zukunft möglich sein, die Herausforderungen, die neue Infektionskrankheiten mit sich bringen, anzunehmen.

Gould schreibt im „Book of the Worms“ zu Recht, dass Mikroben lange vor dem Menschen auf der Erde waren und den Menschen mit Sicherheit überdauern werden. Wir sollten alles tun, um deren Sieg über uns möglichst lange hinauszuschieben.

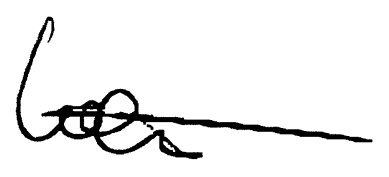

Prof. Dr. T. Welte

\section{Korrespondenzadresse}

\section{Prof. Dr. T. Welte}

Klinik für Pneumologie

Medizinische Hochschule Hannover Carl-Neuberg-Str. 1, 30625 Hannover welte.tobias@mh-hannover.de

\section{Larsen, Ziegenfuß \\ Beatmung}

Heidelberg, Berlin: Springer 2009, 4. Auflage, 453 S., 112 Abb., (ISBN 978-3-540-888116), 44.00 EUR

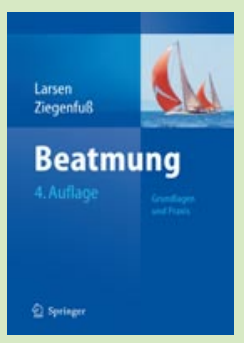

In dem Buch „Beatmung" von Larsen und Ziegenfuß werden die Themen maschinelle Beatmung und Atemunterstützung aus unterschiedlichsten Perspektiven bearbeitet. Das bereits in der 4. Auflage erscheinende Buch ist in den letzten 12 Jahren eine wertvolle Quelle, um Wissen bei der Beatmung von Patienten zu erlangen.

- Zu Beginn werden anatomische und physiologische Grundlagen der Atmungsorgane beschrieben: gut verständlich und in aller Ausführlichkeit, so dass auch der anspruchsvolle Leser bei Bedarf sein Vorwissen verbessern kann und damit einen Fundus für klinische Zusammenhänge erlangt.

- Pathophysiologische Veränderungen bei der respiratorischen Insuffizienz wie z.B. Diffusionsstörungen, erhöhtes Lungenwasser oder Störungen des Lungenkreislaufes bauen darauf auf.

- Alle standardmäßig angewandten Beatmungsformen als auch alternative Verfahren werden plausibel dargestellt. Außerdem sind unkonventionelle Verfahren zur respiratorischen Unterstützung beschrieben.

- Praktische Anleitungen zum Einsatz von Respiratoren oder Airwaymanagement ergänzen theoretische Inhalte durch anwenderorientierte Beispiele. Für den Alltagsgebrauch sind z.B. auch Pharmakotherapie, Dosierungen der üblichen Medikamente als auch diagnostische Methoden beschrieben. Selbst praktische Tipps zu genutzten diagnostischen und therapeutischen Instrumenten sind zu finden.

- Beatmungsprobleme bei intensivmedizinisch relevanten Krankheitsbildern werden im abschließenden Teil beleuchtet. Die Inhalte orientieren sich stets an aktuellen wissenschaftlichen Erkenntnissen.
Im Vorwort bewerten die Autoren das Buch als kompaktes Lehr- und Lernbuch. Dies kann beim Lesen nach den ersten Kapiteln bestätigt werden. Bereits die Grundlagen sind leicht verständlich dargestellt, um das notwendige Wissen zu vermitteln, was zur weiteren Bearbeitung dieses Themenkomplexes anregt und befähigt. Sehr nützlich sind Angaben wichtiger Publikationen zu einzelnen Themen, die am Ende jedes Kapitels thematisch zusammengefasst sind und weiteres Eigenstudium fördern. Praktische Anwenderbeispiele tragen zu einer angenehmen Mischung von Theorie und Praxis bei. Gut übersichtliche Tabellen und Abbildungen sind in fast allen Kapiteln zu finden. An vielen Stellen sind relevante Inhalte herausstechend hervorgehoben. Somit können diese wiederholt und leichter behalten werden. Die Abbildungen sind einfach gehalten, erfüllen jedoch ihren Zweck und ergänzen den Inhalt sinnvoll. An einigen Stellen wären jedoch farbige Originalabbildungen der angewandten Geräte und Hilfsmittel wünschenswert. Insbesondere bei dem Kapitel „Einstellungen des Beatmungsgerätes" oder "Airwaymanagement" könnten diese hilfreich sein. Trotzdem macht das Buch optisch einen guten Eindruck und weckt stets das Interesse des Lesers. Insbesondere Inhalte, die didaktisch abgestimmt, im komplexeren Zusammenhang, wiederholt werden, können im Sinne einer Lernspirale besser behalten werden.

Das Buch „Beatmung“ ist ein wertvolles Kompaktlehrbuch, das mit einer ausgewogenen Mischung an praktischen und theoretischen Inhalten den auf Intensivstationen tätigen Ärztinnen und Ärzten als auch Fachpflegepersonal als sinnvolle Vorbereitung und Begleitung für Ihre Arbeit dienen kann. Das Layout ist ansprechend und entspricht den Anforderungen eines modernen Lehrbuchs. Durch das gewählte Format ist es am unmittelbaren Arbeitsplatz gut zu nutzen und kann Lesern nur empfohlen werden.

Sasa Sopka (Aachen) 